Boise State University

ScholarWorks

$5-26-2006$

\title{
Ground-Penetrating Radar Theory and Application of Thin-Bed Offset-Dependent Reflectivity
}

John H. Bradford

Boise State University

Jacob C. Deeds

ChevronTexaco, ETC-Contractor Acquisition and Processing QC Team 


\title{
Ground-penetrating radar theory and application of thin-bed offset-dependent reflectivity
}

\author{
John H. Bradford ${ }^{1}$ and Jacob C. Deeds ${ }^{2}$
}

\begin{abstract}
Offset-dependent reflectivity or amplitude-variationwith-offset (AVO) analysis of ground-penetrating radar (GPR) data may improve the resolution of subsurface dielectric permittivity estimates. A horizontally stratified medium has a limiting layer thickness below which thin-bed AVO analysis is necessary. For a typical GPR signal, this limit is approximately 0.75 of the characteristic wavelength of the signal. Our approach to modeling the GPR thin-bed response is a broadband, frequency-dependent computation that utilizes an analytical solution to the three-interface reflectivity and is easy to implement for either transverse electric (TE) or transverse magnetic (TM) polarizations. The AVO curves for TE and TM modes differ significantly. In some cases, constraining the interpretation using both TE and TM data is critical. In two field examples taken from contaminated-site characterization data, we find quantitative thin-bed modeling agrees with the GPR field data and available characterization data.
\end{abstract}

\section{INTRODUCTION}

Since the early 1980s, offset-dependent reflectivity, or amplitude-variation-with-offset (AVO) analysis, of seismic data has been used successfully to associate shear- and compressional-wave properties with the presence of oil or natural gas (Ostrander, 1984; Allen and Peddy, 1993; Castagna 1993; Castagna et al., 1993; Bradford et al., 1997). We hypothesize that with careful consideration of the physics and material properties governing electromagnetic wave propagation, similar methodologies may be applied to ground-penetrating radar (GPR) data to make detailed material property measurements of the shallow subsurface.

Over the past decade, several workers have investigated the potential for various applications of GPR AVO analysis. Lehmann (1996) discusses complex reflection coefficients and the effects of conductivity, Bergmann et al. (1998) briefly discuss AVO response in the context of a broader synthetic case study, Reppert et al. (2000) examine the potential for measuring the dielectric permittivity contrast at a boundary using Brewster's angle, and Zeng et al. (2000) present a detailed modeling study considering the effects of varying the Cole-Cole parameters on the AVO response.

The Fresnel reflection coefficient curves (Griffiths, 1989) serve as a starting point in understanding the GPR AVO response. This response depends strongly on the polarization of the incident electric field and the dielectric permittivity contrast at the reflecting boundary. The Fresnel equations are derived assuming a monochromatic EM plane wave incident on a half-space boundary separating two isotropic media. In field studies, all of these assumptions are violated to some degree. In this study, we investigate the GPR AVO response to the presence of a thin bed, with thin bed defined as a layer having thickness less than a wavelength $\lambda$ at the dominant signal frequency. Typical GPR frequencies for groundwatercontaminant studies are $50-100 \mathrm{MHz}$, with corresponding wavelengths on the order of 1-2.5 m. Numerous potential GPR targets may fall into the thin-bed category (e.g., a submeter hydrocarbon layer floating on the water table).

Here we investigate the limits of the Fresnel equations and present a thin-bed model that computes the broadband response via the frequency-dependent three-layer reflectivity. Further, we discuss practical considerations for AVO analysis of field data. Finally, we present two field examples taken from contaminated sites at Hill Air Force Base in Utah and the former Wurtsmith Air Force Base in Michigan.

Manuscript received by the Editor August 5, 2004; revised manuscript received May 3, 2005; published online May 26, 2006.

${ }^{1}$ Boise State University, Center for Geophysical Investigation of the Shallow Subsurface, 1910 University Drive, Boise, Idaho 83725-1536. E-mail: johnb@cgiss.boisestate.edu.

${ }^{2}$ ChevronTexaco, ETC-Contractor Acquisition and Processing QC Team, 4800 Fournace Place, Room W645, Bellaire, Texas 77401. E-mail: eeds@ chevrontexaco.com.

(C) 2006 Society of Exploration Geophysicists. All rights reserved. 


\section{THE THIN-BED PROBLEM IN GPR AVO ANALYSIS}

The two most common modes of GPR data acquisition are transverse magnetic (TM), or end on, and transverse electric (TE), or broadside. In TM acquisition, the transmitting and receiving antennas are colinear and are oriented parallel to the survey profile (Figure 1). In TE acquisition, the antennas are parallel and are oriented perpendicular to the survey profile (Figure 1). In TM mode, the electric field is polarized parallel to the plane of reflection for a downgoing wave incident on a horizontal reflector (Figure 1), whereas in TE mode the electric field is polarized perpendicular to the plane of reflection. The plane of reflection is defined as the ver-

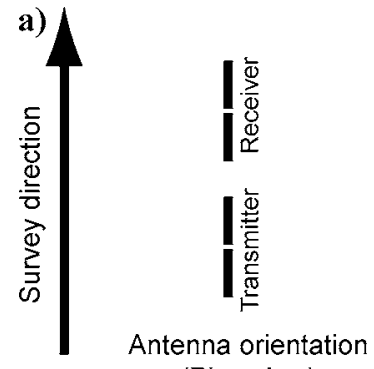

(Plan view)

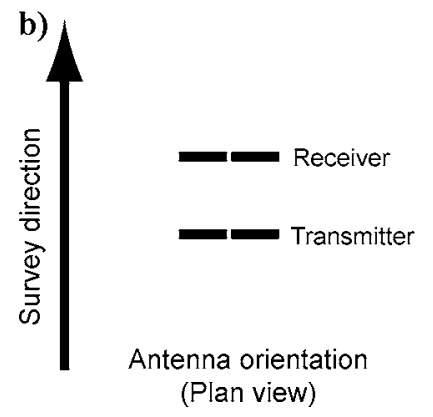

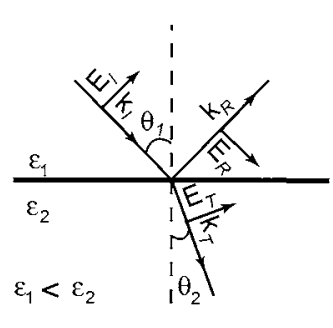

E-field orientation (Inline cross section)

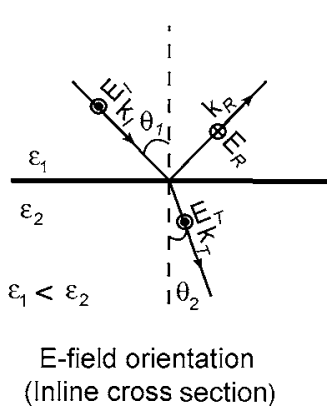

Figure 1. (a) TM and (b) TE configurations for linear dipole antennas and the corresponding electric-field orientations, where $\mathbf{k}$ is the wave vector and $\mathbf{E}$ is the electric field. The subscripts $I, R$, and $T$ indicate incident, reflected, and transmitted fields, respectively.
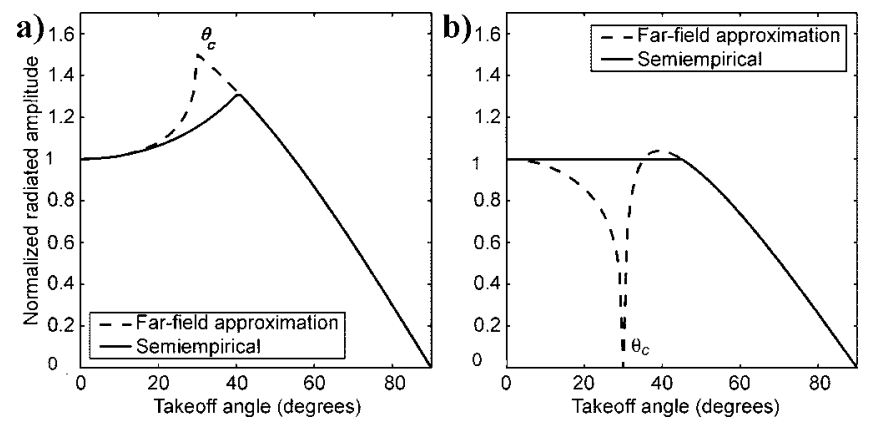

Figure 2. (a) TE and (b) TM radiation patterns for the infinitesimal dipole and semiempirical radiation patterns derived by Bradford (1998); $\theta_{c}$ is the critical angle for an upgoing wave at the earth/air interface. The infinitesimal dipole approximation is typically inconsistent with field observations, while the semiempirical pattern for the TE mode has proven useful in several field and laboratory studies with both shielded and unshielded antennas. The semiempirical pattern for the TM mode has only been consistent with data acquired using shielded antennae. tical plane that bisects the antennas. The mode most commonly used in GPR surveys is TE because more energy is radiated in the TE plane. Because of orthogonal polarizations, the AVO responses of TE and TM modes are dramatically different, and quantifying these differences makes it possible to improve subsurface characterization. In TM mode AVO gradients are larger, and Brewster's angle (Griffiths, 1989; Reppert et al., 2000) can be observed. Because of these attributes, TM data are a more sensitive indicator of permittivity contrasts. However, since less energy is radiated in TM mode (Figure 2), the $\mathrm{S} / \mathrm{N}$ ratio is lower than in TE mode.

For the following analysis, we assume the incident signal is a plane wave in nonconductive, isotropic media. Further, we assume the magnetic permeability is constant and equal to that of free space $\left(\mu=\mu_{0}\right)$ and that the dielectric permittivity $\varepsilon$ is frequency independent. Although these conditions are not generally the case for earth materials, they are reasonable approximations, over the signal bandwidth, for many media in which the GPR signal will propagate efficiently (Annan, 1996).

Consider a three-layer system consisting of an upper and lower half-space (layers 1 and 3) separated by a layer of finite thickness (layer 2). King and Owens (1992) derive the EM plane-wave reflection coefficients $R$ for the more general $n$-layered system, which for the three-layer case reduce to

$$
\begin{gathered}
R_{\mathrm{TE}}=\frac{\gamma_{1}-\gamma_{3}-i\left(\frac{\gamma_{1} \gamma_{3}}{\gamma_{2}}-\gamma_{2}\right) \tan \left(\gamma_{2} l_{2}\right)}{\gamma_{1}+\gamma_{3}-i\left(\frac{\gamma_{1} \gamma_{3}}{\gamma_{2}}+\gamma_{2}\right) \tan \left(\gamma_{2} l_{2}\right)} \\
R_{\mathrm{TM}}=\frac{\gamma_{1} \varepsilon_{3}-\gamma_{3} \varepsilon_{1}-i\left(\frac{\gamma_{1} \gamma_{3} \varepsilon_{2}}{\gamma_{2}}-\frac{\gamma_{2} \varepsilon_{1} \varepsilon_{3}}{\varepsilon_{2}}\right) \tan \left(\gamma_{2} l_{2}\right)}{\gamma_{1} \varepsilon_{3}+\gamma_{3} \varepsilon_{1}-i\left(\frac{\gamma_{1} \gamma_{3} \varepsilon_{2}}{\gamma_{2}}+\frac{\gamma_{2} \varepsilon_{1} \varepsilon_{3}}{\varepsilon_{2}}\right) \tan \left(\gamma_{2} l_{2}\right)},
\end{gathered}
$$

where $l_{2}$ is the thickness of layer 2 . The parameter $\gamma_{n}$ is the vertical component of the wavenumber, given by

$$
\begin{aligned}
\gamma_{n} & =\omega \sqrt{\varepsilon_{n} \mu} \cos \theta_{n} \\
& =\omega \sqrt{\varepsilon_{n} \mu}\left[1-\frac{\varepsilon_{1} \sin ^{2} \theta_{1}}{\varepsilon_{n}}\right]^{1 / 2},
\end{aligned}
$$

where $\omega$ is the angular frequency and $\theta_{n}$ is the angle made by the propagation vector in layer $n$ with respect to vertical. Taking the limit as $l_{2}$ approaches 0 , equations 1 and 2 reduce to the Fresnel equations for reflections from the layer 1/layer 3 boundary. For a finite thickness $l_{2}$, the reflected amplitude is frequency dependent via the $\tan \left(\gamma_{2} l_{2}\right)$ term. The frequency dependence arises because the layer 2/layer 3 reflection interferes with the layer 1/layer 2 reflection. This interference is the source of the thin-bed problem, examined in a classic paper by Widess (1973).

Equations 1 and 2 give the thin-bed amplitude versus incidence angle (AVA) response for a monofrequency signal. The AVO to AVA transform is a function of source-receiver offset and the velocity distribution above the target reflection (Castagna, 1993). Above a limiting layer thickness, the Fresnel equations can be used to predict the AVO behavior for energy reflected from the top of a 
reflectivity sequence. Understanding this limit is critical in planning a data processing and interpretation strategy. The thin-bed reflection amplitude is a function of the signal spectrum, layer thickness, and permittivity contrasts at the layer $1 /$ layer 2 and layer 2/layer 3 boundaries. To compute the reflected field for a broadband signal, we filter the source spectrum using equations 1 or 2 . The time-domain response to the three-layer model is then given by the inverse Fourier transform of the filtered source spectrum.

As an example, we compute the thin-bed AVO response at the layer 1/layer 2 boundary to a range of layer thicknesses $\left(l_{2}\right)$ for a model with $\varepsilon_{1} / \varepsilon_{0}=7, \varepsilon_{2} / \varepsilon_{0}=5, \varepsilon_{3} / \varepsilon_{0}=21$, and a Ricker source wavelet (Figure 3). The measured amplitude is the peak amplitude of the reflection from the layer 1/layer 2 boundary. When $0<$ $l_{2} / \lambda<0.25$, the layer $1 /$ layer 2 reflection is not resolved, and not surprisingly the AVA curves agree poorly with the Fresnel equations. However, when $l_{2} / \lambda \geq 0.5$, the curves are in relatively good agreement. Generalizing this analysis over a broad range of permittivity contrasts, we find that at incidence angles less than $45^{\circ}$, the Fresnel equations are a reasonable approximation of the reflection amplitudes from the top of the reflectivity sequence when $l_{2} / \lambda>0.5-0.75$. A reasonable guideline is that thin-bed analysis should be used when the layer thickness is less than $0.75 \lambda$.

\section{CONSIDERATIONS FOR AVO ANALYSIS OF FIELD DATA}

One approach to GPR AVO data acquisition is to acquire a conventional fixed-offset GPR survey first and then to identify reflectivity anomalies for detailed investigation with limited local common-midpoint (CMP) acquisition. However, some dielectric permittivity configurations that result in a significant AVO anomaly may not produce an obvious reflectivity anomaly in a conventional GPR image (see Hill Air Force Base example below). The method is most effective with continuous CMP profiling throughout the survey area. This allows us to combine adjacent CMPs to improve the $\mathrm{S} / \mathrm{N}$ ratio, enables laterally continuous evaluation of AVO attributes, and provides a statistical basis for error estimates. The drawback to continuous CMP profiling is that current single-channel GPR systems are standard, and multichannel systems designed for CMP acquisition are not commercially available. Single-channel acquisition of CMP data is clearly more laborious and time consuming than conventional fixed-offset data acquisition. However, using a multioffset acquisition methodology similar to that described by Fisher et al. (1992), we refine our field procedures so we can acquire 25-fold data at a rate of about 400-800 common source gathers per day with a three-person crew.

\section{Survey design}

Several factors should be considered in designing AVO acquisition parameters. One of the most important of these is whether sufficient offset is achieved in the survey to observe the AVO effect of interest. Often, the objective requires relatively large angles of incidence $\left(\sim 45^{\circ}\right)$. The required offset is a function of the velocity in the overburden. In many cases, GPR velocities decrease with depth because of increasing moisture content, with a large negative gradient at the transition from the vadose zone to the saturated zone. This velocity decrease causes ray bending toward the vertical. For horizontal layers, this means the incidence angle decreases with depth. Assuming a simple two-layer model with layers of a)
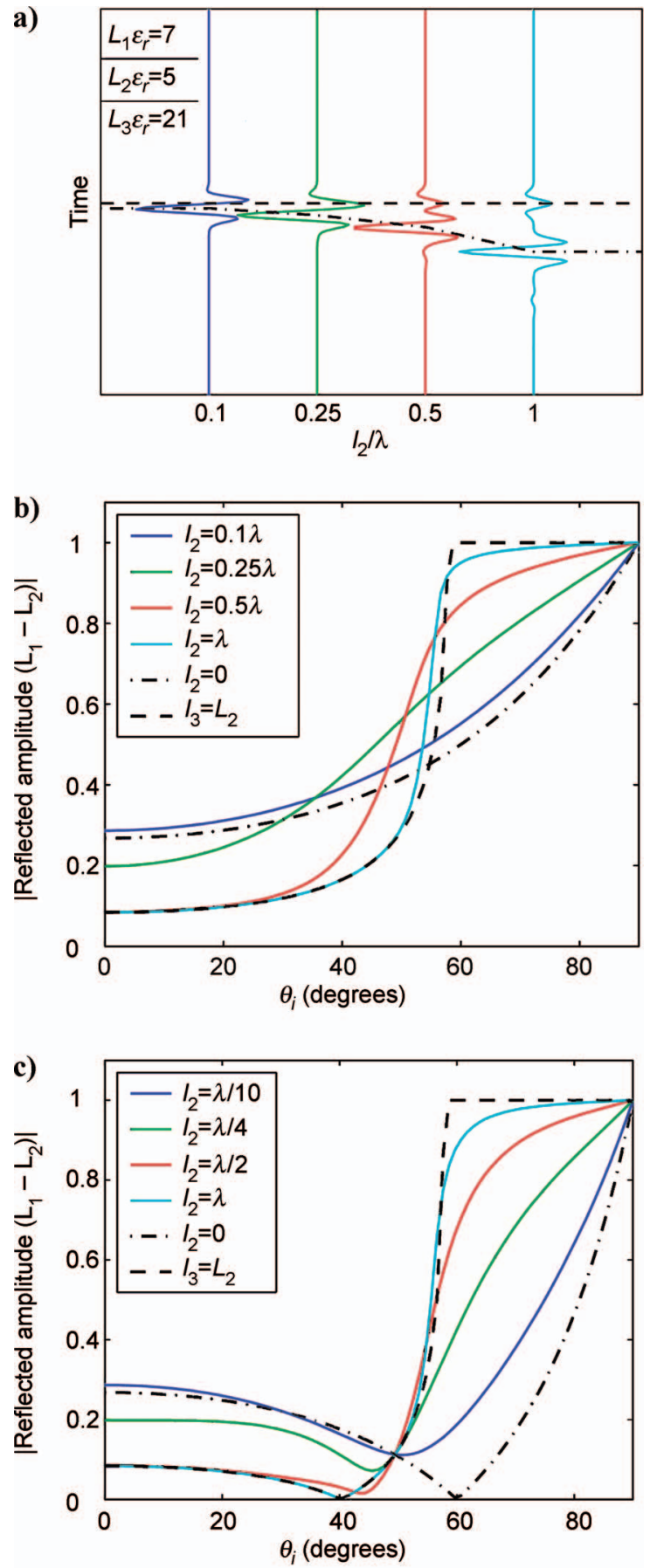

Figure 3. (a) The reflected field from a three-layer model at zero offset with layer thickness varying from $0.1 \lambda$ to $\lambda$. Dashed line indicates the centroid for the layer 1/layer 2 reflection, while the dashed-dotted line shows the centroid for the layer 2/layer 3 boundary. (b) TE reflection amplitude from the layer 1/layer 2 boundary computed using the thin-bed broadband computation. (c) TM reflection amplitude using the thin-bed broadband computation. The $l_{2}=0$ case is the Fresnel equation for the layer 1/layer 3 boundary, and the $L_{3}=L_{2}$ case is the Fresnel equation for the layer 1/layer 2 boundary; $\theta_{i}$ is the incidence angle at the layer 1/layer 2 boundary. The reflections from the upper and lower boundaries are well resolved when $l_{2} / \lambda \geq 0.5$, and the Fresnel equations are a fair approximation of the thin-bed reflection amplitude above this limit. 
thickness $z_{1}$ and $z_{2}$ and the target at the base of the second layer, Snell's law and geometric arguments give the offset $x$ required to reach a given angle of incidence:

$$
\frac{x}{2}=z_{1} \tan \left[\sin ^{-1}\left(\sqrt{\frac{\varepsilon_{2}}{\varepsilon_{1}}} \sin \theta_{2}\right)\right]+z_{2} \tan \theta_{2} .
$$

In this case, $\theta_{2}$ is the incidence angle at the target depth. Additionally, we can derive the maximum angle of incidence that can be reached for a negative velocity gradient using Snell's law and setting $\theta_{1}$ equal to $90^{\circ}$. This yields

$$
\theta_{i}=\sin ^{-1} \sqrt{\frac{\varepsilon_{s}}{\varepsilon_{d}}},
$$

where $\varepsilon_{s}$ and $\varepsilon_{d}$ are the dielectric permittivities at the surface at the target depth, respectively, and $\theta_{i}$ is the incidence angle at the target. Equations 4 and 5 are useful tools in survey design. In most cases a small preliminary study is necessary to estimate site parameters for full-scale survey design.

\section{Amplitude corrections}

Ideally, the processed GPR data amplitudes are a direct measure of the reflection coefficient curve. However, a number of factors affect the recorded amplitude that are unrelated to the reflection coefficient at the target boundary. The recorded or observed amplitude $A_{\text {obs }}$ can be represented as

$$
A_{\text {obs }}=\frac{C_{T} C_{R} P_{T} P_{R} T}{G} \mathrm{Re}^{-\alpha r} A_{\text {sou }},
$$

where $P_{T}$ and $P_{R}$ are the transmitter and receiver antenna radiation patterns, $T$ is the transmission loss across boundaries in the overburden, $G$ is the geometric spreading factor, $R$ is the reflection coefficient, $A_{\text {sou }}$ is the source signal amplitude, $\alpha$ is the attenuation coefficient, $r$ is the length of the travel path, and $C_{T}$ and $C_{R}$ are the transmitter and receiver coupling factors, which are a measure of energy loss at the antenna/earth interface. Each of the parameters in equation 6 may be a function of polarization, ray parameter, frequency, dielectric permittivity, conductivity, and magnetic permeability. Furthermore, the permittivity, conductivity, and permeability are functions of frequency, and the coupling factors are functions of surface roughness. Clearly, the measured amplitude is an extremely complicated function of many variables, most of which are difficult or impossible to determine. However, with a few simplifying assumptions and some understanding of the system we are investigating, we can reduce this complexity to a manageable problem.

First, we assume frequency-independent material properties over the signal bandwidth. Frequency independence is a significant assumption but is reasonable for many materials in which the GPR signal will propagate efficiently (Annan, 1996). Next, we assume that the coupling factors do not vary significantly over the offset range and that the coupling factors and radiation patterns are the same for both the source and receiver. This second assumption is valid over a uniform surface with laterally homogeneous surface material. Finally, we assume transmission losses do not vary significantly with offset. Implicit in this third assumption is that strata in the overburden are flat lying. Using the Fresnel equation for transmission (Griffiths, 1989) to compute combined transmission losses for the up- and downgoing waves, we find that relative transmission losses are less than $5 \%$ for a wide range of subsurface models. For many realistic subsurface models, the $5 \%$ error is valid at angles that are up to $65 \%$ of the critical angle for permittivity decreases and at angles up to $35^{\circ}-55^{\circ}$ for permittivity increases. These limits are not violated for most GPR AVO investigations.

Now, using the above assumptions and taking the ratio of the observed amplitude at any given offset to the amplitude at near offset, we find

$$
\frac{A_{x}}{A_{0}}=\frac{P_{x}^{2} G_{0}}{P_{0}^{2} G_{x}} e^{-\alpha\left(r_{x}-r_{0}\right)} \frac{R_{x}}{R_{0}},
$$

where $P^{2}=P_{T} P_{R}$ and the subscripts $x$ and 0 indicate the given parameter value at offset $x$ or at near offset, respectively. Equation 7 shows that if we evaluate the relative amplitudes along a given reflection, we can eliminate many of the factors that affect the AVO response and that are unrelated to the reflection coefficient. This leaves us computing corrections for the radiation patterns, geometric spreading, and attenuation. While in most cases some or all of the assumptions that go into the derivation of equation 7 are violated to some extent, we have successfully applied this methodology to several field data sets (see field examples below). Our findings suggest that equation 7 provides a reasonable approximation in some cases. Moreover, this is essentially the same set of assumptions that goes into a typical AVO processing scheme in seismic exploration (with the exception of the radiation-pattern correction), where there is a long track record of successful AVO studies (Castagna, 1993).

Radiation-pattern corrections are of particular importance in GPR studies. Many commercial GPR systems use linear dipole antennas, and the radiation patterns have a strong angular dependence. At infinite distance from the source, the antenna appears as an infinitesimal dipole. Engheta et al. (1982) derive a 3D radiation pattern assuming that an infinitesimal horizontal dipole is placed at the boundary of a half-space, with the lower half-space consisting of a low-loss dielectric. These radiation patterns have sharp maxima in TE mode and a null in TM mode at the critical angle of the earth-air interface (Figure 2). In a modeling study, Arcone (1992) demonstrates that the far-field radiation patterns for a linear dipole can differ substantially from the infinitesimal dipole approximation. Additionally, several workers have reported significant deviations from the infinitesimal dipole radiation patterns in the laboratory, even at relatively large distances from the antenna (Annan et al., 1975; Chew and Kong, 1981; Wensink et al., 1990). In particular, the sharp maxima and null points at the critical angle for the earth-air interface are absent or muted in the near field. Wensink et al. (1990) conclude that far-field conditions are not reached by a distance equivalent to $15 \lambda$. In GPR investigations the target is typically within 2-20 $\lambda$ at the characteristic signal frequency. Thus, constructing radiation pattern corrections that do not depend on the far-field approximation is necessary.

In TE mode a semiempirical radiation pattern that at small takeoff angles $\theta_{\mathrm{TO}}$ has the form $1 / \cos \theta_{\mathrm{TO}}$ and then merges with the farfield approximation at large $\theta_{\text {TO }}$ (Figure 2) produces good results for our shielded and unshielded antennas (e.g., Figure 4). This radiation pattern is similar to laboratory measurements given by An- 
nan (1975). We have found the TM radiation pattern more difficult to represent in a simple form. For shielded antennas, a TM radiation pattern with no angular dependence at small $\theta_{\mathrm{TO}}$ that merges with the far-field pattern at large $\theta_{\text {TO }}$ (Figure 2) produces good results in some cases (Bradford, 1998, 2004). In the case of unshielded antennas, we have found significant site and depthdependent variability (Figure 4). Thus, the radiation-pattern correction remains a significant topic of research and a potential source of error. At sites where a background AVO response at the target depth can be measured (i.e., the water-table reflection in an uncontaminated area), taking the ratio of the target AVO curve to the background AVO curve response cancels the radiation-pattern contribution. We have used this approach successfully at several field sites (e.g., the Wurtsmith Air Force Base site discussed below). Ultimately, a solution to radiation-pattern corrections may require case-specific modeling of antenna radiation.

The attenuation correction also requires careful consideration. Separating reflectivity, transmission loss, antenna coupling, and near-surface effects from intrinsic attenuation is difficult. Ideally, we compute the attenuation coefficient using either site-specific lab or field conductivity measurements. However, these measurements are often unavailable, and we are left with typical tabulated values from the literature. If the frequency-independent attenuation assumption is approximately correct over the bandwidth of the signal, then a simple exponential gain correction can be applied. When significant heterogeneity exists in the attenuation structure, such as the transition from the vadose zone to the water-saturated zone, a spatially dependent attenuation correction may be required. We find that for offset-to-depth ratios up to two and attenuation coefficients covering the range from dry to water-saturated sands $(0.01-0.5 \mathrm{~dB} / \mathrm{m}), A_{x} / A_{0}$ in equation 7 is not strongly dependent on the attenuation correction. Given this observation, ignoring the frequency-dependent component of attenuation is reasonable. In cases where the frequency dependence cannot be ignored, approximating the attenuation as a linear function of frequency over the bandwidth of a typical GPR signal is often possible (Turner and
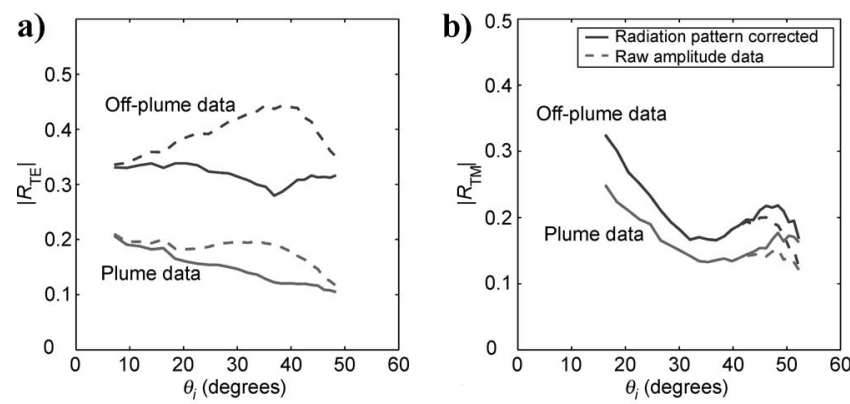

Figure 4. (a) TE and (b) TM amplitude curves taken from a field study at the former Wurtsmith Air Force Base. The amplitude curves are from the water table reflection with CMPs acquired over a hydrocarbon plume (low-amplitude curves) and from an uncontaminated area (high-amplitude curve). In the raw data, the radiation patterns are apparent with a consistent maxima (TE) or minima (TM) present in both the on-plume and off-plume reflections, despite having different reflection amplitudes and AVA curves. Applying a radiation-pattern correction using the semiempirical curves shown in Figure 2 effectively removes the radiationpattern contribution from the TE data but is not effective for the TM data. The infinitesimal dipole approximation is not consistent with either the TE or TM data.
Siggins, 1994). Under these conditions, the spectral ratio or frequency-shift methods may hold promise for calculating a frequency-dependent attenuation correction (Bradford and Wu, 1997; Liu et al., 1998).

\section{Amplitude measurement}

The potential for processing artifacts is important to consider when measuring field-data amplitudes. Typically, surface noise, including the direct waves through the ground and air, travels at a moveout velocity greater than that of subsurface reflections and little interference occurs over a large range of incidence angles. The low-frequency transient usually prevalent within two wavelet cycles of the source has a significant effect on the AVO curve of shallow reflections. However, this energy is present at the low end of the useable frequency spectrum. Often, only bandpass filtering and amplitude recovery corrections are required prior to performing AVO analysis. In general, when soil conditions are such that reflections can be obtained from the target zone, GPR data have relatively high $\mathrm{S} / \mathrm{N}$ ratios and are well suited for AVO analysis. However, the potential for processing or noise artifacts should be considered carefully on a case-by-case basis.

Although the simplifying assumptions presented in the preceding section provide a first-order approximation, we often observe some phase rotation and wavelet dispersion related to complex, frequency-dependent material properties (Olhoeft, 1987). To eliminate the potential for amplitude artifacts associated with phase rotation, we extract wavelet amplitudes from the peak of the envelope function (Sheriff, 2002). Consider a set of Gabor wavelets (Gaussian modulated sine functions) that have the same amplitude but whose phase rotations vary from 0 to $\pi$ (Figure 5). The wavelets have identical power spectra, but the peak values of the wavelets in the time domain show a significant phase dependence (Figure 5). However, the maximum amplitude of the envelope function is independent of phase and is equivalent to the maximum amplitude of the zero-phase wavelet. Using the envelope function as a basis for AVO analysis minimizes the potential for phase-related artifacts.

The amplitude we use for AVO analysis is the local maxima of the envelope function within a time gate bounding a reflection event. To avoid NMO stretch artifacts, we extract amplitudes in the pre-NMO CMP domain. Quantitive analysis of phase information has the potential to provide additional material property information but is beyond the scope of this study.

\section{Comparison of GPR and seismic AVO analysis}

Although the physics for GPR and elastic-wave propagation are different, the general concepts of AVO analysis are the same for both methods. This includes the generalized set of factors that can affect measured amplitudes summarized by Castagna (1993). The discussion of the previous sections addresses factors that may require additional emphasis in GPR data analysis. Two additional points of contrast simplify GPR AVO analysis relative to seismic data. First, radar typically has only the direct wave through the ground, which interferes with reflections at large offsets, whereas seismic reflection has direct wave modes in addition to ground roll, guided waves, etc., that interfere with reflections in both near and far-offset ranges. Thus, in GPR data there is typically a much broader window in which reflections are recorded without interference from other modes. Second, for many earth materials, EM 
wave propagation has a stronger frequency dependence than elastic wave propagation (Turner and Siggins, 1994). However, in seismic AVO studies the signal typically travels tens or hundreds of wavelengths prior to reaching the target, while in GPR studies the target depths are often on the order of ten wavelengths or less. Much shorter propagation distances, relative to the GPR wavelength, result in decreased potential for wave dispersion to develop and greater potential to resolve heterogeneity in the overburden. Given these observations, GPR AVO studies may have similar or better success than exploration seismic AVO studies.

\section{FIELD EXAMPLES: DIRECT DETECTION OF NAPL}

Of the many potential applications for GPR AVO analysis, detection of nonpolar organic liquid contaminants has received particular attention (Baker, 1998; Bergmann et al., 1998; Deeds, 2002; Bradford, 2003, 2004). These contaminants are collectively referred to as nonaqueous-phase liquids (NAPLs) because of their low solubility in water. NAPLs are subcategorized by their density relative to water; dense NAPLs (DNAPL) are denser than water, and light NAPLs (LNAPL) are lighter than water. It follows that

a)

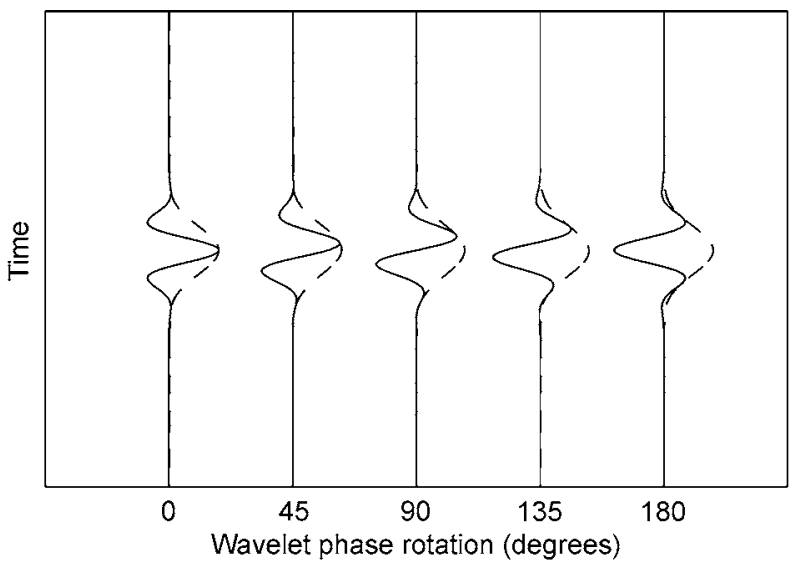

b) 2

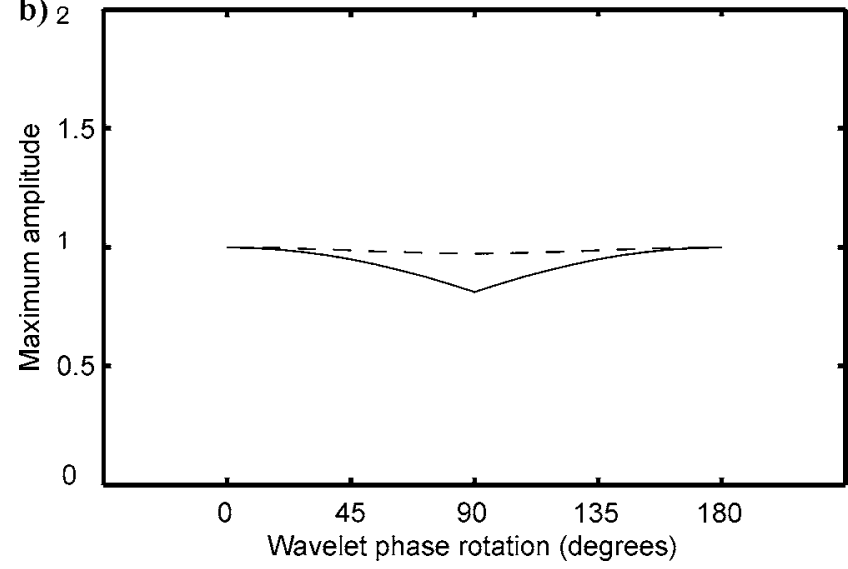

Figure 5. (a) Wavelets with the same amplitude but variable phase rotation. (b) the peak wavelet value and peak value of the envelope function versus phase rotation. The dashed line is the envelope function, and the solid line is the wavelet. The envelope function is independent of phase rotation and therefore provides a good basis for AVO analysis.
DNAPLs tend to sink through the water column until they reach a low-permeability layer, whereas LNAPLs tend to remain near the water table with free product floating on the water column and residual product present in a smear zone above and below the water table. Both LNAPLs and DNAPLs leave a zone of residual contamination along their migration routes. Chlorinated solvents (DNAPL) and fuel hydrocarbons (LNAPL) are common examples.

NAPLs typically have low relative permittivity $\left(\varepsilon / \varepsilon_{0} \sim 2.5\right)$ and low electric conductivity $(\sigma \sim 0.01-0.1 \mathrm{mS} / \mathrm{m})$ relative to freshwater $\left(\varepsilon / \varepsilon_{0} \sim 80, \sigma \sim 0.5 \mathrm{mS} / \mathrm{m}\right)$, which makes them attractive targets for characterization using electrical geophysical methods. As the NAPL displaces water in the sediment pore space, a zone of anomalous electrical properties may be induced. A firstorder conceptual model is based on the premise that NAPL-contaminated zones will have low permittivity and low conductivity relative to the surrounding formation. A number of controlled-spill experiments are consistent with this model (DeRyck et al., 1993; Brewster and Annan, 1994; Campbell et al., 1995; Bradford, 2004). This model has also proven effective in the interpretation of geophysical data acquired over a fresh LNAPL spill (Orlando, 2002) and over aged LNAPL and DNAPL spills at another field site (Lien and Enfield, 1998; Newmark et al., 1998; Deeds and Bradford, 2002). However, aged LNAPL spills at some sites have anomalously high electric conductivity (Monier-Williams, 1995; Sauck et al., 1998; Atekwana et al., 2000). This divergence stems from the incorrect assumption that the electric properties of the unaltered LNAPL control the electric properties of the contaminated zone. Sauck (1998) proposes a conceptual model that explains the high-conductivity LNAPL plume in terms of increased dissolvedsolids concentrations in the groundwater related to biogenic activity. In either case, GPR AVO analysis can be a useful tool for contaminated-zone characterization under the following two conditions: (1) A permittivity anomaly is associated with the contaminated zone, and (2) the zone boundary is sufficiently sharp relative to the signal wavelength to generate a reflection.

Here we demonstrate quantitative thin-bed AVO analysis at two LNAPL-contaminated sites. A key objective in this work is minimizing complexity in the processing stream while producing quantitive data interpretation that is consistent with field observations.

\section{Hill Air Force Base}

At Operable Unit 1 (OU-1), Hill Air Force Base (HAFB), Utah, disposal of a diverse mixture of contaminants from 1952-1973, including lubricating oils, fuels, and solvents, resulted in a large NAPL plume. This plume is currently estimated at 20,000 gallons covering about 7 acres. The plume is comprised almost entirely of LNAPL; the primary constituent is weathered jet fuel. The maximum thickness of free NAPL is around $0.3 \mathrm{~m}$. Both free and residual phase NAPL are present in a roughly $1.5-\mathrm{m}$-thick smear zone above the water table. Annual water-table fluctuations of 1-2 m control the smear-zone thickness. As the water table rises, buoyant NAPL smears upward; as the water table falls, the contaminant pools under the effect of gravity.

Surficial sediments at the site are comprised of the Provo alluvium, which consists of 6-10 m of silt, sand, and gravel in a cutand-fill stratigraphy. The alluvium is underlain by the Alpine Formation, a thick, silty clay unit that acts as an aquitard about which the local water table fluctuates seasonally.

In previous work at HAFB, Lien and Enfield (1998) found that 
NAPL-contaminated soils in the vadose zone at OU-1 had anomalously low electric conductivity. Their finding that NAPL at the site is associated with an electrical property anomaly coupled with favorable results of previous GPR imaging work at HAFB (Young and Sun, 1996, 1998) led us to select OU-1 for a field research location.

\section{Data acquisition and initial interpretation}

In October 2000 we acquired a $2973-\mathrm{m}^{2} 3 \mathrm{D}$ survey at OU-1 designed to test the feasibility of detecting LNAPL-contaminated zones using multioffset GPR methodologies. The data consisted of 20 parallel, multioffset TE GPR profiles acquired with $50-\mathrm{MHz}$ antennas. Additional acquisition details are listed in Table 1. We placed the survey area along the periphery of the known contaminant plume with the intention of surveying from contaminated to uncontaminated sediments.

Data quality was excellent, with a well-defined reflection from the surface of the Alpine clay present throughout the survey area as well as reflections within the Provo alluvium (Figure 6). We measured the $3 \mathrm{D}$ velocity distribution using prestack depth migration (PSDM) velocity analysis (Deeds and Bradford, 2002). In the interpretation of the PSDM data volume and velocity model, we identified a high-velocity anomaly approximately $8 \mathrm{~m}$ below a datum located within a topographic low along the alluvium/clay contact (Figure 6). The reflection originating from the upper surface of the velocity anomaly forms a dome-shaped structure above the topographic low. The maximum thickness of the high-velocity zone is about $1.5 \mathrm{~m}$, which is $0.36 \lambda$ at the data's dominant frequency of $35 \mathrm{MHz}$.

\section{AVO analysis and results}

The target for this analysis is the reflection from the top of the high-velocity anomaly. For AVO preprocessing, we applied corrections for geometric spreading, radiation pattern, and exponential attenuation. We computed path length, takeoff angle, and incidence angle, assuming straight rays. This computation gave incidence angles ranging from $11^{\circ}-45^{\circ}$ for the reflection from the top of the highvelocity anomaly. We applied the radiation-pattern correction using the semiempirical TE function shown in Figure 2. The input relative permittivity at the ground surface was 5.7, which we computed from the velocity of the direct ground wave $(0.13 \mathrm{~m} / \mathrm{ns})$. For the attenuation correction, we used a frequencyindependent attenuation coefficient and adjusted the value so the reflection amplitudes from the clay layer remained approximately constant with increasing offset. We believe this approach is reasonable in this case because the signal is being reflected from the boundary between relatively high-velocity vadose-zone alluvium and very-low-velocity water-saturated clay. Under these conditions, and given the range of incidence angles for the survey geometry, the Fresnel

Table 1. Data-acquisition parameters at Hill Air Force Base.

Parameter Value

\begin{tabular}{ll}
\hline Survey type & TE, 3D, constant azimuth \\
GPR system & Sensors and software Pulse EKKO, \\
& 100A 50-MHz unshielded \\
antennae
\end{tabular}

Survey size

$61 \mathrm{~m} \times 49 \mathrm{~m}$ (inline $\times$

crossline)

Minimum/maximum offset $1.8 / 9.1 \mathrm{~m}$

Common source fold 25

Source interval $\quad 0.61 \mathrm{~m}$

Receiver interval $\quad 0.30 \mathrm{~m}$

Crossline spacing $\quad 2.4 \mathrm{~m}$

Sampling interval $\quad 1.6 \mathrm{~ns}$

Recording time $\quad 500 \mathrm{~ns}$

Stacks/source 16

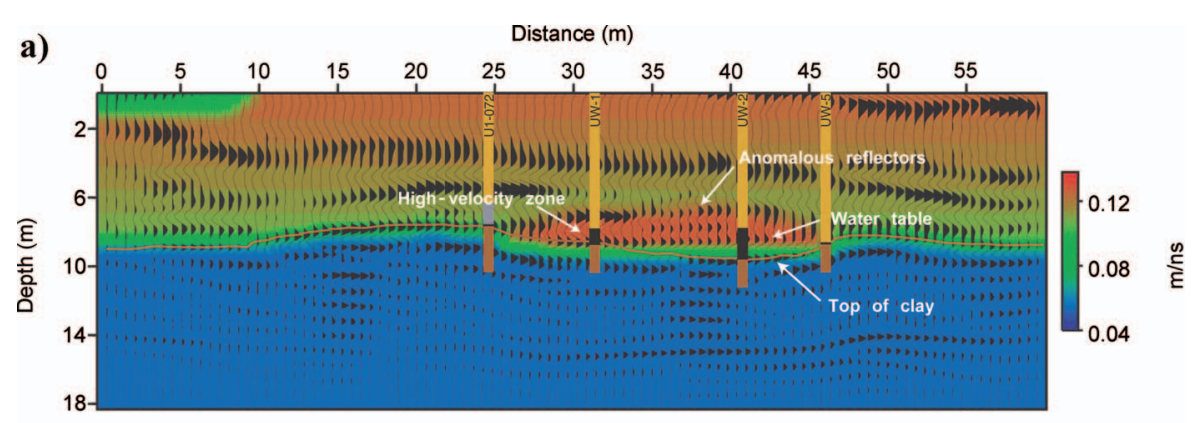

b)

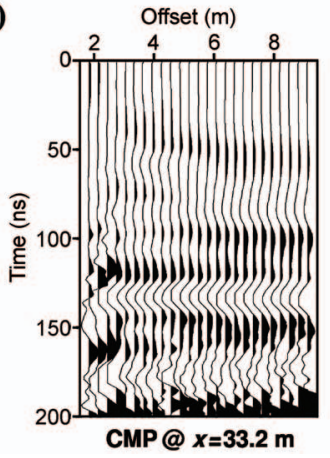

c)

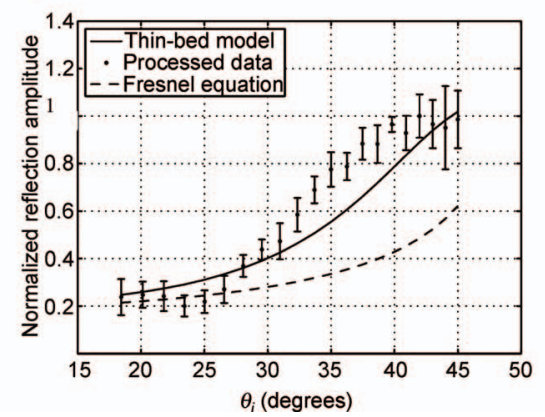

Figure 6. (a) PSDM velocity model with migrated trace overlay. We identified a high-velocity zone within a topographic low on the clay surface. In a subsequent coring effort, hydrocarbon contamination was found within this zone (contaminated interval in black). (b) NMOcorrected, true-amplitude CMP gather at $33.2 \mathrm{~m}$. (c) Reflection amplitudes from the top of the high-velocity anomaly taken from 20 CMPs centered on the UW-1 core. The amplitude of the reflection from the top of the high-velocity zone $(\sim 100 \mathrm{~ns})$ shows a clear increase in amplitude with increasing offset. We only used offsets of less than $8 \mathrm{~m}$ in the quantitative analysis to minimize artifacts related to weak interference of the direct wave through the ground at far offsets. While the Fresnel equation predicts the general trend of the data, the thin-bed computation, based on equation 1 and the PSDM velocity model, is in much closer agreement with the field observations. 
equations predict an approximately flat AVO curve. The computed attenuation coefficient is $3.1 \mathrm{~dB} / \mathrm{m}$, which is at the low end of the range given for silts by Davis and Annan (1989).

The reflection amplitude from the top of the high-velocity anomaly increases significantly with increasing offset (Figure 6). Using the broadband computation based on equation 1, with a 35$\mathrm{MHz}$ Ricker wavelet and the permittivity model estimated from PSDM velocity analysis, we find the thin-bed model produces a curve that correlates with the field data. Also, the trend is qualitatively consistent with the Fresnel equation computation, which predicts increasing amplitude with offset.

\section{Discussion}

In this field study, we derived a subsurface permittivity model using velocity analysis and then predicted the offset-dependent reflectivity using thin-bed analysis, finding it to be consistent with the field data. In cores acquired subsequent to our GPR survey, elevated levels of hydrocarbon (varying from 1\%-4\% total volume) were found within the high-velocity zone (Deeds and Bradford, 2002). So, in addition to demonstrating the methodology successfully, we located a previously unidentified zone of LNAPL contamination from the associated dielectric permittivity anomaly. These results strongly suggest that the GPR AVO method has potential as an exploratory tool in contaminated-site characterization.

\section{Wurtsmith Air Force Base (AFB)}

This former fire training facility, designated FT-02, is located on the now-decommissioned Wurtsmith AFB in Oscoda, Michigan. In the early 1990s, a free NAPL plume was identified. The plume resulted from incomplete burning of large quantities of jet fuel during training exercises. At that time, the free-phase plume was up to $0.3 \mathrm{~m}$ thick and extended more than $180 \mathrm{~m}$ downgradient from FT02 (Bermejo et al., 1997; Sauck et al., 1998).

Table 2. Data-acquisition parameters at the former Wurtsmith Air Force Base.

\begin{tabular}{ll} 
Parameter & Value \\
\hline Survey type & $\begin{array}{l}\text { TE, TM, 3D, constant } \\
\text { azimuth }\end{array}$ \\
GPR system & $\begin{array}{l}\text { Sensors and software, } \\
\text { Pulse EKKO 100A 100-MHz } \\
\text { unshielded antennas }\end{array}$ \\
Survey size & $30 \mathrm{~m} \times 30 \mathrm{~m}$ (inline $\times$ \\
crossline)
\end{tabular}

The stratigraphy below the site consists of fine- to mediumgrained sand and gravel deposits extending approximately $20 \mathrm{~m}$ deep. Below this unit is a 6-30-m-thick silty clay layer, thought to be the lower boundary for contaminant migration. The water table in the unconfined aquifer was 3-5 $\mathrm{m}$ below the surface at the time of our investigation.

Central to the selection of this site for the current study is a series of geophysical investigations carried out by Sauck et al. (1998) and Bermejo et al. (1997). They found the site provided excellent conditions for GPR with strong reflections well below the water table. Additionally, they found a well-defined, high-attenuation anomaly coincident with the LNAPL plume. Through resistivity and self-potential measurements and by inference from the GPR data, they concluded that high electrical conductivity was associated with both the LNAPL and the dissolved-phase plumes.

By the time of our survey, remediation activities had significantly reduced the total NAPL volume, and free NAPL was no longer found in monitoring wells. We assume that residual-phase NAPL remains in a zone above the water table with a distribution similar to that occupied by the previously existing free-phase plume. Critically, the water table at the time of our investigation was at approximately the same depth as during the previously reported geophysical investigations (Bermejo et al., 1997; Sauck et al., 1998). Thus, it is reasonable to use the older characterization data as a measure of relative fluid distribution.

\section{Data acquisition and initial interpretation}

For this analysis, we present results from a representative 122$\mathrm{m}$ profile taken from a larger $3 \mathrm{D}$ multioffset survey. The data were acquired with 100-MHz antennas in both TE and TM modes. Additional acquisition parameters are given in Table 2. The stacked section is of excellent quality (Figure 7) and shows many of the same features noted by Sauck et al. (1998). The most prominent reflection originates from the water table at around $60 \mathrm{~ns}$. Qualitatively, the plume attenuation anomaly appears as a zone of muted reflection amplitudes below the water table and extending to depth. A quantitative measure of this effect is the full-trace amplitude (FTA), which is the sum of the envelope function (Sheriff, 2002) along a given trace. The plume location is clearly delineated as a relatively sharp FTA trough (Figure 7). The profile extends well beyond the east and west boundaries of the attenuation anomaly and is roughly centered on the plume. In the off-plume areas, strong reflections are evident to greater than 500 ns. Sauck et al. (1998) interpret the deep (>150 ns), hummocky reflections as paleodunes.

Detailed NMO velocity analysis reveals no apparent lateral velocity anomaly that correlates with the attenuation anomaly. The approximately $1 \mathrm{D}$ velocity structure has a relatively high-velocity vadose zone $(\sim 0.135 \mathrm{~m} / \mathrm{ns})$ and a sharp drop to water-saturatedsand velocity $(\sim 0.065 \mathrm{~m} / \mathrm{ns})$ across the water table (Figure 7$)$.

\section{AVO analysis and results}

The target of this analysis is the water-table reflection since it is the primary boundary controlling the NAPL distribution. The AVO preprocessing flow includes bandpass filtering (12-25-200-400 $\mathrm{MHz}$ ), a geometric spreading correction, and a laterally variable attenuation correction.

To estimate the attenuation coefficients in the vadose zone, we first compute the rms values of vadose-zone resistivity measured 
by Sauck et al. (1998) for the on-plume and off-plume areas. We then use the resistivity values to compute the attenuation coefficient with the equation for low-loss media given by Davis and Annan (1989). This gives attenuation coefficients of 0.26 and $0.066 \mathrm{~dB} / \mathrm{m}$ for the on-plume and off-plume areas, respectively. Because frequency dependence is not included, this approach gives the minimum attenuation coefficient, but the estimates are reasonably close to tabulated values ranging from dry to water-saturated sand (Davis and Annan, 1989).

Using the straight-ray assumption to compute the offset-to-incidence-angle transform, the acquisition geometry (Table 2) yields incidence angles at the water table ranging from $8^{\circ}-49^{\circ}$ in $\mathrm{TE}$ mode and $16^{\circ}-52^{\circ}$ in TM mode.

After applying spreading and attenuation corrections, the data clearly show the antenna directivity, with well-developed lobate patterns that have the same shape in on-plume and off-plume areas (Figure 4). This suggests that antenna directivity does not vary significantly along the profile. Given this observation and the fact that we have a good basis for computing the background water-table-amplitude response - a long section of high $\mathrm{S} / \mathrm{N}$ data outside the plume boundaries — we avoid the radiation-pattern correction problem by taking the ratio of the anomalous amplitudes to a background AVO curve. This computation cancels the radiation-pattern contribution, thereby minimizing the potential for radiationpattern artifacts. In this case we define the background AVO curve by taking the average AVO response of 112 adjacent CMPs from an area outside the plume attenuation anomaly (Figure 7).

$\mathrm{AVO}$ anomalies are not obvious from qualitative interpretation of the CMP gathers (Figure 8). However, quantitative comparison of the plume and background data reveals well-defined TE and TM AVO anomalies that correlate with the plume attenuation anomaly (Figure 9). The anomalous zone correlates with a decrease in the near-offset water-table reflection amplitude of as much as $40 \%$. In TE mode, the water-table reflection amplitude within the plume anomaly tends to decay more rapidly than the background amplitudes (Figure 9). The TM data show the opposite trend; on-plume amplitudes grow larger with increasing offset relative to the background AVO curve.

For thin-bed analysis, we compute the broadband, offset-dependent reflectivity for a range of models using the measured thickness (Bermejo et al., 1997; Sauck et al., 1998) of the LNAPL zone above the water table $(0.3 \mathrm{~m})$ for the plume permittivity structure and a simple half-space model for the background permittivity structure. Here we assume the transition from the vadose zone to the water-saturated zone occurs over an interval that is much less than the wavelength of the signal. Given that the signal wavelength is approximately $1.6 \mathrm{~m}$ at the dominant frequency of $\sim 80 \mathrm{MHz}$ and that the transition from the vadose zone to full saturation may occur over a few centimeters in coarse-grained sands (Bedient et al., 1994), we believe the half-space background model plume AVO response. is a reasonable approximation. A model with an intermediate thinlayer permittivity of $\varepsilon_{\mathrm{TB}} / \varepsilon_{0}=8.5$ in the plume area produces amplitude curves that agree well with both the TE and TM field data (Figure 9).

Figure 7. (a) CMP stack, (b) velocity model averaged over the 3D volume, and (c) total trace carbon plume is evident where there is a decrease in total trace amplitude between 190 and $235 \mathrm{~m}$. The water table reflection is at approximately $60 \mathrm{~ns}$. We used the interval from 240-255 $\mathrm{m}$ to define the background AVO curve and CMPs from 205-215 $\mathrm{m}$ to define the
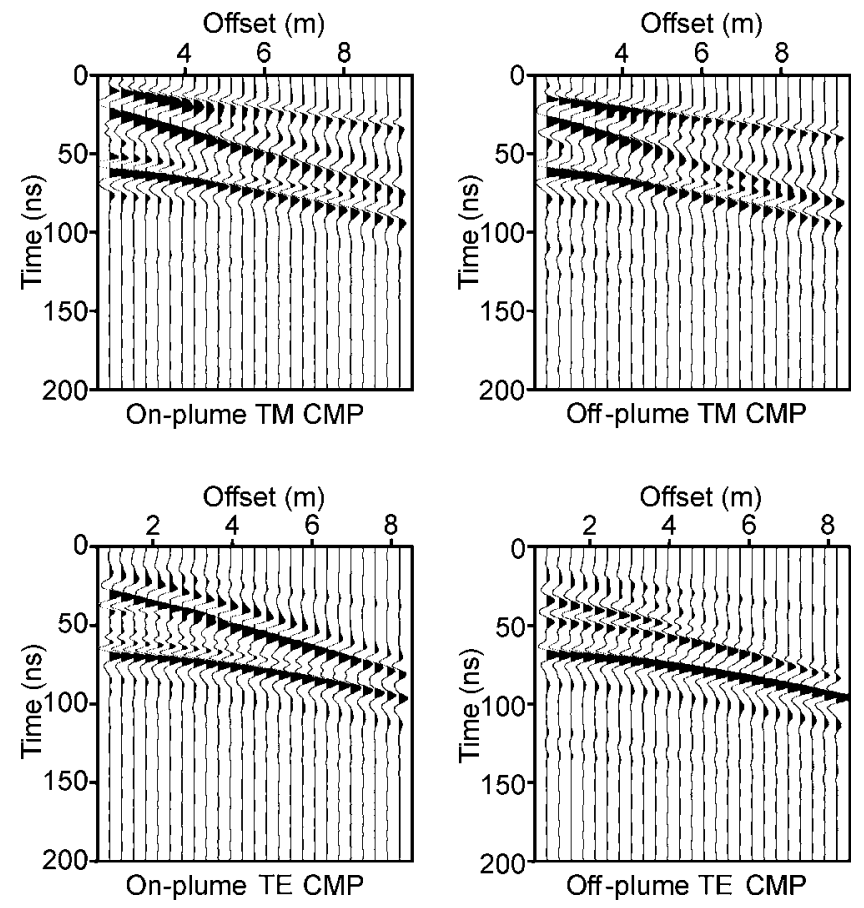

Figure 8. Representative gathers from the on-plume and off-plume areas. The water table reflection is approximately $60 \mathrm{~ns}$. AVO anomalies are not obvious from qualitative inspection of the gathers. 
a)

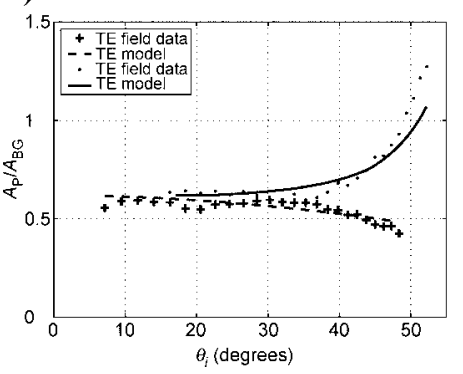

b)

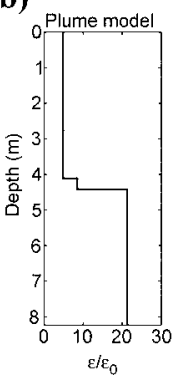

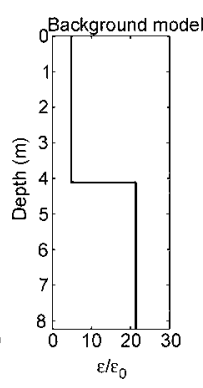

Figure 9. (a) Plume anomaly $\left(A_{\mathrm{P}}\right)$ to background $\left(A_{\mathrm{BG}}\right)$ amplitude ratios for TE and TM modes. See Figure 8 for the range of onplume and off-plume CMPs used in this computation. Average standard deviations of the ratios are 0.12 and 0.13 for TM and TE modes, respectively. (b) Plume and background permittivity models used to compute the model ratios, which agree well with the field data.

\section{DISCUSSION}

When the velocity decreases across a reflecting boundary, the Fresnel equations predict little variability in the AVO trend for both TE and TM modes over a large range of velocity contrasts (Bradford, 2003). The lack of lateral variability in the long-wavelength velocity structure at FT-02 indicates that the NAPL or biogeochemical process which produces an electric conductivity anomaly (1) does not produce a permittivity anomaly or (2) has a permittivity anomaly below the resolution of stacking velocity analysis. These observations suggest that a water-table AVO anomaly is unlikely at this site. However, we observed well-defined TE and TM water-table AVO anomalies coincident with the known plume location. If only TE data were acquired, where increased decay of the AVO curve is observed within the plume, we might attribute the anomaly to an underestimate of the attenuation coefficient within the contaminate-altered vadose zone. However, this is inconsistent with the TM data, which show an opposite trend. A thin-bed model based on the available characterization data is consistent with all of the observations and including the TM data is critical in constraining the interpretation.

Two scenarios could result in the zone of intermediate permittivity: (1) The contaminant has low permittivity, and a mixed LNAPL/water zone decreases the permittivity in the capillary fringe and upper part of the saturated zone; or (2) the LNAPL zone has anomalously high permittivity, increasing the vadose-zone permittivity just above the water table. The 0.3-m-thick NAPL-contaminated zone is below the resolution of a conventional reflection image. That is, the zone is thinner than $\lambda / 4$ at the dominant signal frequency of $80 \mathrm{MHz}$. With this limitation we cannot differentiate between scenario 1 and scenario 2 . Yet the presence of the thin layer produces significant, measurable amplitude effects that we may use to increase the detail in our subsurface characterization.

\section{CONCLUSIONS}

Multifold GPR profiling coupled with quantitative analysis of GPR offset-dependent reflectivity is a valuable tool that can improve the detail and accuracy of GPR subsurface characterization. Thus far the method has seen limited application, probably in large part because of the difficulty of collecting continuous multifold data with commercially available single-channel GPR systems.

With future hardware advances we expect that multifold acquisition will become more efficient and more widely used.

The Fresnel equations provide a starting point in understanding the GPR AVO response, but the assumptions that go into their derivation are violated in many field situations. We have presented an easily implemented broadband model of the three-layer reflection coefficients to address the thin-bed problem in GPR AVO analysis. In most cases when the thin bed is greater than $0.75 \lambda$, the Fresnel equations reasonably approximate the reflection amplitudes from the top of the reflectivity sequence; below this limit, investigators should use thin-bed analysis.

By making simplifying assumptions such as constant antenna coupling, constant radiation pattern, and frequency-independent material properties, the GPR AVO response is reduced to a tractable problem. These assumptions in some cases appear to reasonably approximate field conditions. We have also found that coincident TE and TM data allow us to take advantage of the polarization dependence of the reflection coefficients to limit the nonuniqueness in data interpretation. With careful processing, quantitative GPR AVO analysis of field data is possible and holds significant potential as an exploratory tool.

\section{ACKNOWLEDGMENTS}

The U. S. Department of Energy funded this work under the Environmental Management Science Program, grant DE-FG0799ER15008. Boise State University acknowledges support of this research by Landmark Graphics Corp. via the Landmark University Grant Program.

\section{REFERENCES}

Allen, J. L., and C. P. Peddy, 1993, Amplitude variation with offset: Gulf Coast case studies: Geophysical Development Series vol. 4, SEG.

Annan, A. P., 1996, Transmission dispersion and GPR: Journal of Environmental and Engineering Geophysicists, 0, 125-136.

Annan, A. P., W. M. Waller, D. W. Strangway, J. R. Rossiter, J. D. Redman, and R. D. Watts, 1975, The electromagnetic response of a low-loss, 2-layer, dielectric earth for horizontal electric dipole excitation: Geophysics, 40, 285-298.

Arcone, S. A., 1992, Numerical studies of the radiation patterns of resistively loaded dipoles: Journal of Applied Geophysics, 33, 39-52.

Atekwana, E. A., W. A. Sauck, and D. D. Werkema Jr., 2000, Investigations of geoelectrical signatures at a hydrocarbon contaminated site: Journal of Applied Geophysics, 44, 167-180.

Baker, G. S., 1998, Applying AVO analysis to GPR data: Geophysical Research Letters, 25, 397-400.

Bedient, P. B., H. S. Rifai, and C. J. Newell, 1994, Ground water contamination: Transport and remediation: Prentice-Hall, Inc.

Bergmann, T., J. O. A. Robertsson, and K. Holliger, 1998, Finite-difference modeling of electromagnetic wave propagation in dispersive and attenuating media: Geophysics, 63, 856-867.

Bermejo, J. L., W. A. Sauck, and E. A. Atekwana, 1997, Geophysical discovery of a new LNAPL plume at the former Wurtsmith AFB, Oscoda, Michigan: Groundwater Monitoring and Remediation, 17, 131-137.

Bradford, J. H., 1998, Characterizing shallow aquifers with wave-propagation based geophysical techniques: Imaging and attribute analysis: Ph.D. dissertation, Rice University.

- , 2003, GPR offset-dependent reflectivity analysis for characterization of a high-conductivity LNAPL plume: Symposium on the Application of Geophysics to Environmental and Engineering Problems (SAGEEP), Environmental and Engineering Geophysical Society, Proceedings, 238-252.

, 2004, 3D Multi-offset, multi-polarization acquisition and processing of GPR data: A controlled DNAPL spill experiment: Symposium on the Application of Geophysics to Environmental and Engineering Problems (SAGEEP), Environmental and Engineering Geophysical Society, Proceedings, 514-527.

Bradford, J. H., D. S. Sawyer, and C. A. Zelt, 1997, AVO analysis of lowvelocity, shallow sands $(<50 \mathrm{~m})$ : 67th Annual International Meeting, 
SEG, Expanded Abstracts, 158-162.

Bradford, J. H., and Y. Wu, 1997, Time-frequency representation of seismic signals via matching pursuit decomposition with complex Ricker wavelets: Fall meeting, AGU, EOS Abstracts Supplement, 33.

Brewster, M. L., and A. P. Annan, 1994, Ground-penetrating radar monitoring of a controlled DNAPL release: $200 \mathrm{MHz}$ radar: Geophysics, 59, $1211-1221$.

Campbell, D. L., J. E. Lucious, K. J. Ellefson, and M. Deszcz-Pan, 1995, Monitoring of a controlled LNAPL spill using ground-penetrating radar: Symposium on the Application of Geophysics to Environmental and Engineering Problems (SAGEEP), Environmental and Engineering Geophysical Society, Proceedings, 511-517.

Castagna, J. P., 1993, AVO analysis - Tutorial and review, in J. P. Castagna and M. M. Backus, eds., Offset-dependent reflectivity - Theory and practice of AVO analysis: Investigation in Geophysics, SEG, 3-36

Castagna, J. P., M. L. Batzle, and T. K. Kan, 1993, Rock physics — The link between rock properties and AVO response, in J. P. Castagna and M. M. Backus, eds., Offset-dependent reflectivity - Theory and practice of AVO analysis: Investigations in Geophysics, SEG, 135-171.

Chew, W. C., and J. A. Kong, 1981, Electromagnetic field of a dipole on a two-layer earth: Geophysics, 46, 309-315.

Davis, J. L., and A. P. Annan, 1989, Ground-penetrating radar for highresolution mapping of soil and rock stratigraphy: Geophysical Prospecting, 37, 531-551.

Deeds, J. C., 2002, Amplitude variation with offset (AVO) of ground penetrating radar data for detection of non-aqueous phase liquids: M.S. thesis, University of Wyoming.

Deeds, J. C., and J. H. Bradford, 2002, Characterization of an aquitard and direct detection of LNAPL at Hill Air Force Base using GPR AVO and migration velocity analysis: Ninth International Conference on Ground Penetrating Radar, International Society for Optical Engineering, Proceedings, 323-329.

DeRyck, S. M., J. D. Redman, and A. P. Annan, 1993, Geophysical monitoring of a controlled kerosene spill: Symposium on the Application of Geophysics to Environmental and Engineering Problems (SAGEEP), Environmental and Engineering Geophysical Society, Proceedings, $5-19$

Engheta, N., C. H. Papas, and C. Elachi, 1982, Radiation patterns of interfacial dipoles: Radio Science, 17, 1557-1566.

Fisher, E., G. A. McMechan, and A. P. Annan, 1992, Acquisition and processing of wide-aperture ground-penetrating radar data: Geophysics, 57 495-504.

Griffiths, D. J, 1989, Introduction to electrodynamics: Prentice-Hall, Inc.

King, R. W. P., and M. Owens, 1992, Lateral electromagnetic waves: Theory and applications to communications, geophysical exploration, and remote sensing: Springer-Verlag New York, Inc.

Lehmann, F., 1996, Fresnel equations for reflection and transmission at boundaries between conductive media, with applications to georadar problems: 6th International Conference on Ground-Penetrating Radar, International Society for Optical Engineering, Proceedings, 555-560.

Lien, B. K., and C. G. Enfield, 1998, Delineation of subsurface hydrocarbon contaminant distribution using a direct push resistivity method:
Journal of Environmental and Engineering Geophysicists, 2, 173-179.

Liu, L., J. W. Lane, and Y. Quan, 1998, Radar attenuation tomography using the centroid frequency downshift method: Journal of Applied Geophysics, 40, 106-116.

Monier-Williams, M., 1995, Properties of light non-aqueous phase liquids and detection using commonly applied shallow sensing geophysical techniques: Symposium on the Application of Geophysics to Environmental and Engineering Problems (SAGEEP), Environmental and Engineering Geophysical Society, Proceedings, 1-15.

Newmark, R. L., W. D. Daily, K. R. Kyle, and A. L. Ramirez, 1998, Monitoring DNAPL pumping using integrated geophysical techniques: Journal of Environmental and Engineering Geophysicists, 3, 7-13.

Olhoeft, G. R., 1987, Electrical properties from $10^{-3}$ to $10^{9} \mathrm{~Hz}-$ Physics and chemistry: 2nd International Symposium on the Physics and Chemistry of Porous Media, American Institute of Physics, Proceedings, 281298.

Orlando, L., 2002, Detection and analysis of LNAPL using the instantaneous amplitude and frequency of ground-penetrating radar data: Geophysical Prospecting, 50, 27-41.

Ostrander, W. J., 1984, Plane-wave reflection coefficients for gas sands at nonnormal angles of incidence: Geophysics, 49, 1637-1648.

Reppert, P. M., F. D. Morgan, and M. N. Toksöz, 2000, Dielectric constant determination using ground-penetrating radar reflection coefficients Journal of Applied Geophysics, 43, 189-197.

Sauck, W. A., 1998, A conceptual model for the geoelectrical response of LNAPL plumes in granular sediments: Symposium on the Application of Geophysics to Environmental and Engineering Problems (SAGEEP), Environmental and Engineering Geophysical Society, Proceedings, 805817

Sauck, W. A., E. A. Atekwana, and M. S. Nash, 1998, High conductivities associated with an LNAPL plume imaged by integrated geophysical techniques: Journal of Environmental and Engineering Geophysicists, 2, 203-212.

Sheriff, R. E., 2002, Encyclopedic dictionary of applied geophysics: SEG.

Turner, G., and A. F. Siggins, 1994, Constant Q attenuation of subsurface radar pulses: Geophysics, 59, 1192-1200.

Wensink, W. A., G. Greeuw, J. Hofman, and J. K. Van Deen, 1990, Measured underwater near-field e-patterns of a pulsed, horizontal diplot antenna in air: Comparison with the theory of the continuous wave, infinitesimal dipole: Geophysical Prospecting, 38, 805-830.

Widess, M. B., 1973, How thin is a thin bed?: Geophysics, 38, 1176-1180.

Young, R. A., and J. Sun, 1996, 3D ground penetrating radar imaging of a shallow aquifer at Hill Air Force Base, Utah: Journal of Environmental and Engineering Geophysicists, 1, 97-108.

1998, Extracting a radar reflection from a cluttered environment using 3-D interpretation: Journal of Environmental and Engineering Geophysicists, 3, 121-131.

Zeng, X., G. A. McMechan, and T. Xu, 2000, Synthesis of amplitude versus offset variations in ground-penetrating radar data: Geophysics, 65 113-125. 